\begin{tabular}{|c|c|}
\hline Title & Photodegradation mechanisms in laser dyes: A laser irradiated ESR study \\
\hline Author(s) & Yamashita, Mikio; Kashiwagi, Hiroshi \\
\hline Citation & $\begin{array}{l}\text { IEEE Journal of Quantum Electronics, 12(2), 90-95 } \\
\text { https://doi.org/10.1109/JQE.1976.1069096 }\end{array}$ \\
\hline Issue Date & $1976-02$ \\
\hline Doc URL & http:/hdl.handle.net/2115/45309 \\
\hline Rights & $\begin{array}{l}\text { () } 1976 \text { IEEE. Personal use of this material is permitted. However, permission to reprint/republish this material for } \\
\text { advertising or promotional purposes or for creating new collective works for resale or redistribution to servers or lists, } \\
\text { or to reuse any copyrighted component of this work in other works must be obtained from the IEEE. }\end{array}$ \\
\hline Type & article \\
\hline File Information & JQE12-2_90-95.pdf \\
\hline
\end{tabular}

Instructions for use 


\title{
Photodegradation Mechanisms in Laser Dyes: A Laser Irradiated ESR Study
}

\author{
MIKIO YAMASHITA AND HIROSHI KASHIWAGI, MEMBER, IEEE
}

\begin{abstract}
The photodegradation mechanisms in xanthene dye-laser solutions are investigated at $77 \mathrm{~K}$ by the direct detection of a decrease in the concentration of dye molecules in the lowest excited triplet state $\left(T_{1}\right.$ state) and of free radicals produced after degradation using an ESR technique under UV $(3511+3638 \AA)$ or $5145-\AA$ laser irradiations. It is shown that the ESR spectral shape of the radicals induced by an UV laser beam is quite different from that of those induced by a $5145-\AA$ beam. For those radicals, the irradiation time and power dependences, the effects of dye and solvent molecular structures and the role of the $T_{1}$ state by the addition of cyclooctatetraene (COT) quenchers are examined in some detail. As a result the two following laser-induced photochemical reactions are proposed: in UV laser- or flashlamp-pumped dye lasers, the $\mathrm{C}-\mathrm{H}$ bond rupture of a solvent molecule due to the energy transfer of a dye molecule in a higher excited triplet state, which is produced by a $T-T$ absorption of a dye molecule in the $T_{1}$ state, results in a radical and a leuco compound of the dye On the other hand, in the case of the 5145-A laser excitation, a partially reversible change of a dye molecule in the $T_{1}$ state produced by only a one-photon absorption results in another radical which depends on the chromophoric structure of the dye.
\end{abstract}

\section{INTRODUCTION}

$\mathrm{P}$ HOTODEGRADATION of organic dyes severely limits the lifetime of dye lasers, but the degradation mechanism is not yet well understood. Until now the studies on the degradation have been performed by detecting changes in the absorption and fluorescence spectra of dye-laser solutions before and after photodegradation [1]-[3]. In this paper we report the investigations on the photodegradation mechanism of xanthene laser dyes by a different method, in which the behaviors of the laser-induced free radicals and dye molecules in the lowest excited state $\left(T_{1}\right.$ state) at $77 \mathrm{~K}$ are monitored directly, using an ESR technique during and after UV ( $3511+$ $3638 \AA$ ) or visible (from 5145 to $4545 \AA$ ) laser irradiations [4]. Our method has the following advantages: 1) the dye molecules in the $T_{1}$ state and the free radicals occurring after degradation, photochemically produced, are directly detected;2) the sensitivity to the paramagnetic products, induced by laser irradiations, is much higher; 3 ) as a result of cooling the ethanolic dye solution to $77 \mathrm{~K}$, the solution becomes glassy [5], [6] and the products are stabilized at the irradiation point by being trapped in the rigid glass. On the other hand, because of the high intensity, wide tunability, and nar row spectral width of the pumping laser light, studies on the laser-induced photochemical reactions will be performed very often in the future and will exert significant influence on the field of photochemical physics.

At first we show that two different free radicals are induced

Manuscript received May 30, 1975; revised September 15, 1975.

The authors are with the Radio- and Opto-Electronics Division, Electrotechnical Laboratory, Tanashi, Tokyo, Japan. in an R6G-ethanol solution by using either UV or visible laser irradiations. They differ in their ESR spectral shape according to the different wavelengths, and by increasing the laser power the amount of each increases, while the intensity of the $\Delta m= \pm 2$ ESR spectrum of the dye molecules in the $T_{1}$ state decreases. After that, the dependence of the radical concentration on the irradiation time and power, the effects of molecular structures of some laser dyes and solvents on the producing processes of radicals, and the role that the $T_{1}$ state of the dye molecules plays in the photochemical reactions are investigated. Finally, the laser-induced reaction sequences in the case of an UV laser irradiation and in that of a $5145-\AA$ laser irradiation, which are quite different from each other, are proposed.

\section{EXPERIMENTAL}

Each dye-rhodamine 6G (R6G), rhodamine B (RB), rhodamine $S(R S)$, acridine red (AR), acridine orange (AO), and fluorescein disodium salt (FDS), all produced by the Eastman Kodak or Tokyo Kasei Kogyo Companies-was dissolved in ethanol, methanol, or dimethylsulfoxide (DMSO) at $1 \times$ $10^{-3}-\mathrm{M}$ concentration. We used Kanto Chemical's guaranteed reagent-grade ethanol, Tokyo Kasei's guaranteed reagentgrade methanol, and spectra-grade DMSO. The COT molecules, obtained from the Aldrich Chemical and Tokyo Kasei Kogyo Companies, were used as a triplet quencher for the R6G dye. In order to determine the photodegradation mechanism easily, the oxygen-free solution, outgassed by a freeze-thaw process, was sealed in a 4-mm-O.D. quartz tube. The experimental arrangement is nearly identical to the one we used previously to investigate the different behaviors of dye molecules in the $T_{1}$ state during laser excitation [4], [6]-[8]. Both the ESR spectrum of the $H_{\min }$ line corresponding to $\Delta m= \pm 2$ transitions in dye molecules in the $T_{1}$ state [4] and that of the free radicals, induced by laser light, were recorded with a Varian E-4 spectrometer with an optical transmission cavity. The peak-to-peak heights of the radical signal and of the $H_{\min }$ line were used in a first approximation to calculate the concentrations of the radicals and of the dye molecules in the $T_{1}$ state, respectively. The $H_{\min }$ line was recorded by using such a low-power $5145-\AA$ laser beam $\left(0.29, \mathrm{~W} / \mathrm{cm}^{2}\right)$ that no measurable amount of radicals occurred. The solution, cooled by inserting it into liquid nitrogen in a quartz Dewar, was irradiated by an unfocused beam consisting of UV $(3511+3638 \AA)$ light or $5145-\AA$ light of an Ar-ion laser (except for the AO dye solution, which was irradiated by a $4880-\AA$ beam). The transmittances of the quartz tube without th dye solution, which was inserted into the Dewar vessel containing liquid nitrogen, were measured for UV and $5145-\AA$ laser beams. The results were that the attenuations of the laser 
irradiation power due to the air, the liquid nitrogen, and the cylindrical quartz sheets of the Dewar vessel and the sample tube were about 35 and 45 percent, and the beam diameters were about 3.2 and $3.5 \mathrm{~mm}$ for the UV and $5145 . \AA$ laser beams, respectively.

\section{Results AND Discussions}

In both cases-UV $(3511+3638 \AA)$ and $5145-\AA$ laser irradiations to the $R 6 \mathrm{G}$ in an ethanol solution at $77 \mathrm{~K}$-an ESR spectrum in the free-radical region was observed, which grew in intensity while the $\Delta m= \pm 2$ spectrum of the dye molecule in the $T_{1}$ state decreased in intensity. The spectral shape of the radical (denoted by FR1) which appeared in the dye solution during or after the UV laser irradiation, was the same as that appearing during or after irradiation by a high-pressure $\mathrm{Hg}$ lamp, but was quite different from that of the radical (denoted by FR2) which appeared during or after the $5145 . \AA$ laser irradiation, as shown in Fig. 1 . In the case of an Ar-ion laser irradiation in the blue-green region with a shorter wavelength than $5145 \AA$, the same spectral shape as that of the FR2 was always observed. The ESR spectrum of the FR1 obtained in the R6G-ethanol solution is quite good in comparison with that obtained in a hydrogen-peroxide-ethanol solution by Gibson et al. [9] using the photolysis method with a low-pressure $\mathrm{Hg}$ lamp. They assigned the hyperfine pattern to the ethanol radical, $\mathrm{CH}_{3} \dot{\mathrm{C}} \mathrm{HOH}$, formed by an $\alpha$-hydrogen abstraction from ethanol. Therefore, the FR1 spectrum appearing in the oxygen-free solution is considered to be due to the $\mathrm{CH}_{3} \dot{\mathrm{C}} \mathrm{HOH}$ radical which arises by the abstraction of a hydrogen atom of an ethanol molecule by the laser-excited R6G molecule. On the other hand, the precise

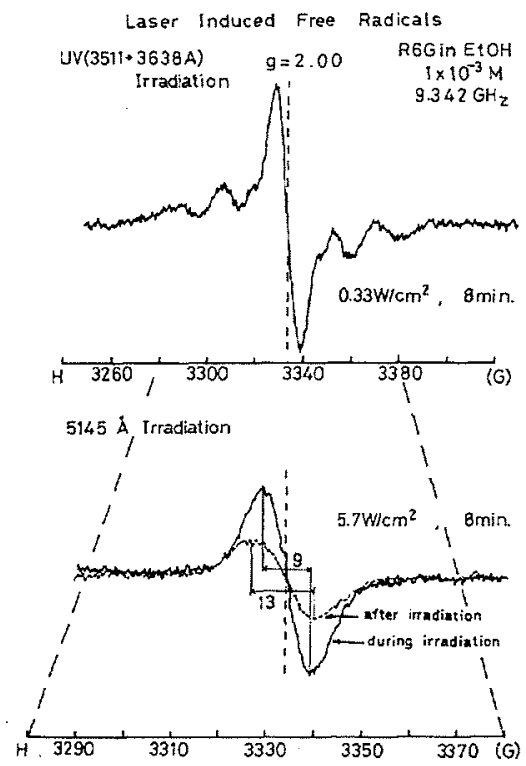

Fig. 1. Observed frequency-derivative ESR spectra of the free radical FRI in a $1 \times 10^{-3}-\mathrm{M} \mathrm{R} 6 \mathrm{G}$-ethanol solution at $77 \mathrm{~K}$ after UV laser irradiation (upper spectrum) and of the free radical FR2 during (solid line of the lower spectra) or after (dotted line of the lower ones) a $5145-\AA$ laser irradiation. The $U V$ and $5145-\AA$ laser irradiation time and power are $8 \mathrm{~min}$ and $0.33 \mathrm{~W} / \mathrm{cm}^{2}$, and $8 \mathrm{~min}$ and 5.7 $W / \mathrm{cm}^{2}$, respectively origin of the FR2 radical induced by the $5145-\AA$ laser irradiation cannot be stated at present. However, there seems to be no doubt that the radical depends on the chromophoric structure of the R6G dye, which will be mentioned subsequently.

At first, for the cases of UV and 5145- $\AA$ laser irradiations to the R6G-ethanol solution, the dependence of the peakto-peak heights of the radical and triplet signals on the increase of the repetition number of the irradiation, using a constant time $(2 \mathrm{~min})$ and a constant power $\left(0.53 \mathrm{~W} / \mathrm{cm}^{2}\right.$ for $\mathrm{UV}$ and $3.4 \mathrm{~W} / \mathrm{cm}^{2}$ for $\left.5145 \AA\right)$ was examined. Therefore, the spectra of the FR1 or the FR2 with the corresponding wavelengths were recorded, and afterwards the $H_{\text {min }}$ signal which was used to calculate the concentration of the dye molecules in the $T_{1}$ state was recorded during a $5145-\AA$ laser irradiation with such a weak power that no FR2 could increase. From the results shown in Figs. 2 and 3, we find that the increase in the concentration of the radicals is correlative with the decrease in the concentration of dye molecules in the $T_{1}$ state in the cases of the FR1 and FR2. This is considered to be due to the following causes: 1) the shortening of the triplet-state lifetime is due to the paramagnetic interaction between the induced radicals and the dye molecules in the $T_{1}$ state; and 2) the

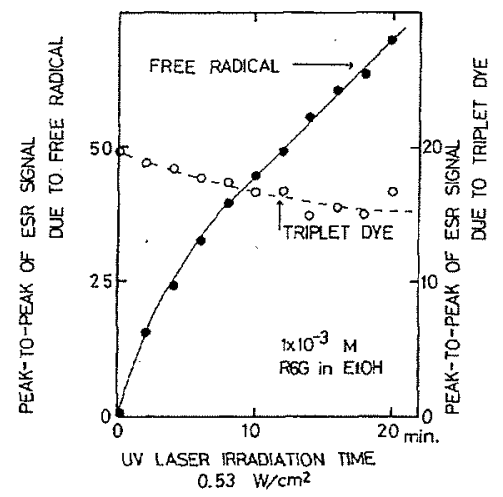

Fig. 2. Peak-to-peak heights of the FRI radical spectrum (symbol induced after an UV laser irradiation and of the $H_{\min }$ line (symbol o) in an R6G-ethanol solution versus the repetition number $(2 \times$ $n \mathrm{~min})$ of the irradiation using a fixed power $\left(0.53 \mathrm{~W} / \mathrm{cm}^{2}\right)$ for 2 min. The $H_{\min }$ line corresponding to $\Delta m= \pm 2$ transitions in the $T_{1}$ state was recorded during a $5145-\AA$ laser irradiation such a low power $\left(0.29 \mathrm{~W} / \mathrm{cm}^{2}\right)$ that no other radical was produced.

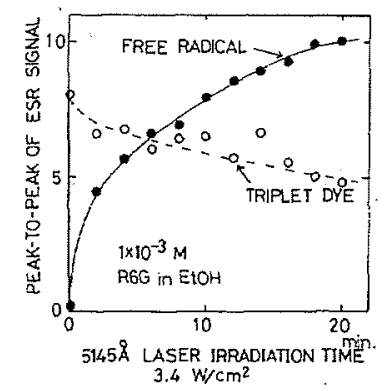

Fig. 3. Peak-to-peak heights of the FR2 radical spectrum induced after a 5145- $\mathrm{A}$ laser irradiation and of the $H_{\min }$ line in an R6Gethanol solution versus the repetition number $(2 \times n \mathrm{~min})$ of the irradiation using a fixed power $\left(3.4 \mathrm{~W} / \mathrm{cm}^{2}\right)$ for $2 \mathrm{~min}$. The $H_{\min }$ line was recorded in the same way as in the case of Fig. 2. The symbols - and o correspond to those in Fig. 2. 
decrease of the effective dye molecules is due to the change of its molecules into free radicals. But, on the basis of the experimental result that the triplet-state lifetime did not change significantly before and after UV or $5145 . \AA$ laser irradiations, it seems that the correlation between the increase of free radicals and the decrease of dye molecules in the $T_{1}$ state is mainly due to the last point.

Secondly, the variation of the concentration of the radicals was examined by increasing the irradiation power during a constant time $(2 \mathrm{~min}$ for an UV irradiation and $5 \mathrm{~min}$ for a $5145-\AA$ irradiation). The dependences of the peak-to-peak heights of the FR1 and FR2 on the irradiation power are shown in Fig. 4. This figure shows that the curve of the FR1 induced by an UV laser irradiation increases with the square of the irradiation power, while the one of the FR2 induced by a $5145-\AA$ laser irradiation starts to increase linearly and then begins to saturate with increasing power. This saturation curve is similar to that of the concentration of dye molecules in the $T_{1}$ state depending on the pumping laser power [7]. Thus we can find that the FR1 and FR2 are produced by quite different optical processes, by two sequential single-photon absorptions including a $T-T$ absorption and by a singlephoton absorption, respectively. The fact that the $T-T$ absorption is related to the producing process of the FR1 supports the foregoing assignment that the FRI is a $\mathrm{CH}_{3} \dot{\mathrm{CHOH}}$ radical, because it is energetically sufficient to rupture the $\mathrm{C}-\mathrm{H}$ bonds of ethanol by an energy transfer from the $\mathrm{R} 6 \mathrm{G}$ molecules in a higher triplet state, which is produced by a stepwise single-photon absorption or a $T-T$ absorption of a dye molecule in the $T_{1}$ state, to ethanol molecules [10], [11]. Furthermore, the comparison between the concentration dependence of the FRI on the UV laser irradiation power and that of the FR2 on the $5145-\AA$ laser irradiation power indicates that the $F R 1$ is produced more easily by two orders in the production rate per unit power and unit time.

In order to investigate more thoroughly the effects of dyes and organic solvents on the producing processes of free radicals, we recorded the ESR spectra of those radicals occurring in dye-ethanol solutions for different dyes (R6G, RB, RS, AR, $A O$, and FDS) and occurring in R6G-organic solvents for different organic solvents (EtOH, MeOH, and DMSO). As shown in Figs. 5 and 6 , the spectral shapes of the FR1 induced by the UV laser irradiation depend remarkably on the molecular structure of the solvents but only a little on that of the laser dyes. This also supports the previously mentioned conclusion that the FR1 is due to the solvent decomposition by the laserexcited dye molecule. On the other hand, the spectral shapes of the FR2 induced by the $5145-A$ laser irradiation (only the AO dye solution was irradiated by a 4880 - $\AA$ laser beam) depend more on the chromophoric structure of the dyes than on the molecular structure of the solvents, which is shown in Figs. 7 and 8. In the case of the visible laser irradiation the FR2 radical spectra of the dye solutions differ in the half-width only if their chromophoric structure is different. However, all the radicals we obtained have almost the same $g$-value of $g=2.00$. Also it was evident that the FR1 or the FR2 in the $\mathrm{AO}$ - and FDS-ethanol and in the R6G-dimethylsulfoxide solutions were easily induced compared with those radicals induced in the other dye-ethanol and R6G-solvent solutions, respectively. Furthermore, it was observec for all the examined dyes that the signal intensities of the FR2 after the irradiation cutoff rapidly decreased to constant intensities when we used the visible laser irradiation, while those of the

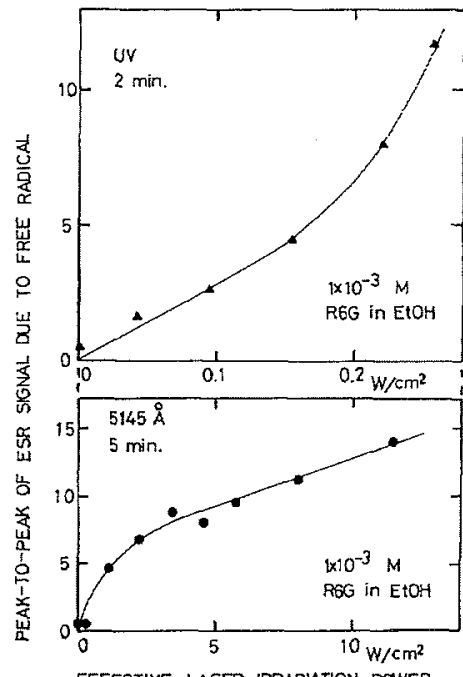

Fig. 4. Variation of the peak-to-peak heights of the FR1 and FR2 radical spectra induced in an R6G-ethanol solution after UV (upper curve) and 5145-A (lower curve) laser irradiations as a function of the irradiation power for a constant time ( 2 and 5 min for UV and $5145-\AA$ laser irradiations, respectively). 


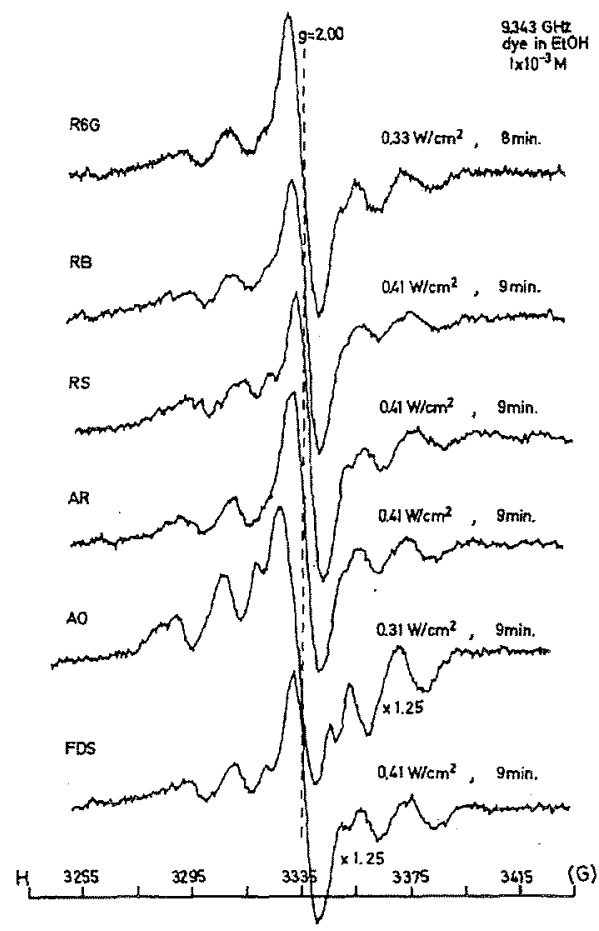

Fig. 5. Observed frequency-derivative ESR spectra of free radicals in duced after an UV laser irradiation at $77 \mathrm{~K}$ for different dyes dissolved in ethanol.

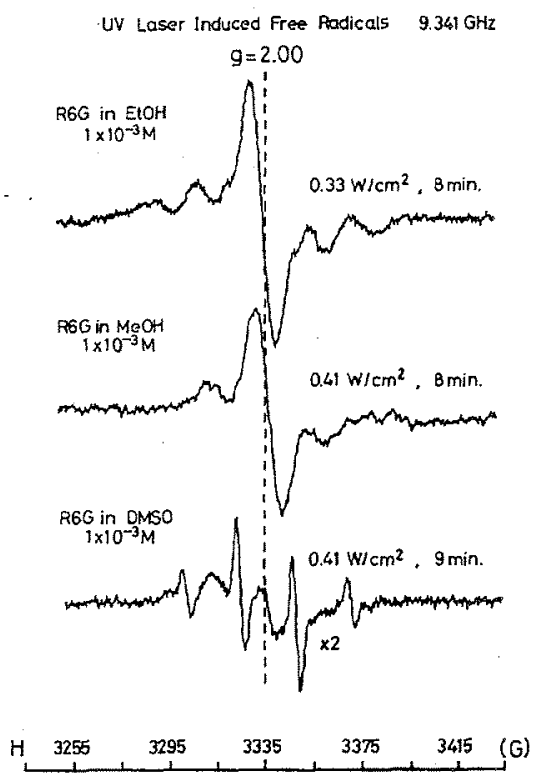

Fig. 6. Observed frequency-derivative ESR spectra of free radicals induced after an $U V$ laser irradiation at $77 \mathrm{~K}$ for $\mathrm{R} 6 \mathrm{G}$ in different solvents.

FR1 using the UV laser irradiation did not change. The decay times of the signal intensities of the FR2 in the R6G-ethanol solution at $77 \mathrm{~K}$ are about $1.0 \mathrm{~s}$, which is a little less than the triplet-state lifetime of the solution [7]. This suggests that the

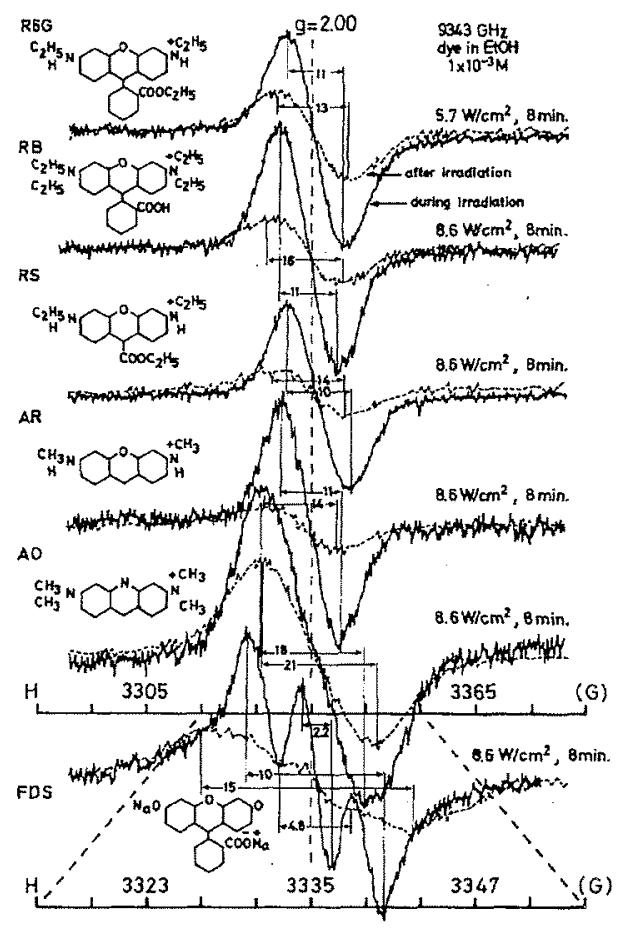

Fig. 7. Observed frequency-derivative ESR spectra of free radicals induced during (solid line) and after (dotted line) a 5145-A laser irradiation at $77 \mathrm{~K}$ for different dyes dissolved in ethanol. Spectral half-widths in gauss units and dye molecular structures are also noted.

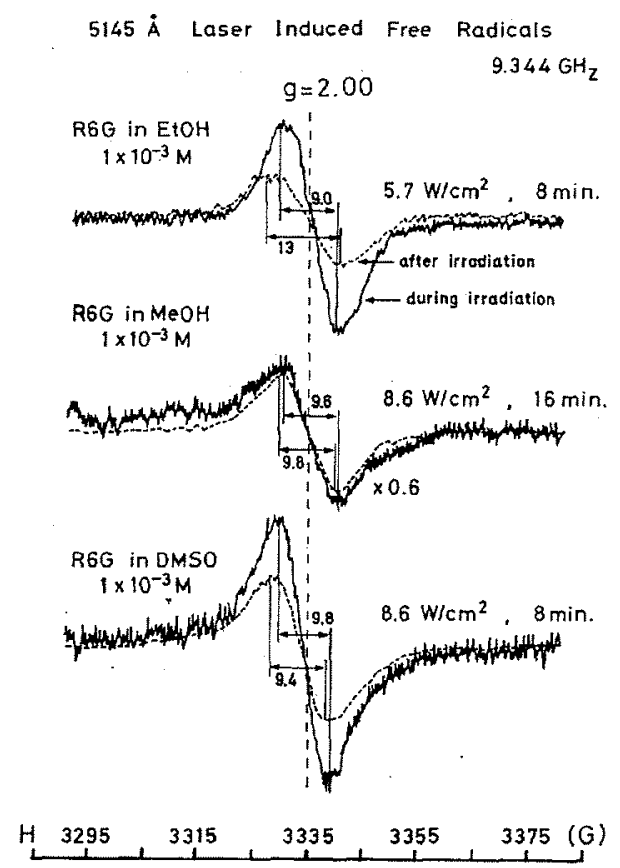

Fig. 8. Observed frequency-derivative ESR spectra of free radicals induced during (solid line) and after (dotted line) a 5145- $A$ laser irradiation at $77 \mathrm{~K}$ for $\mathrm{R} 6 \mathrm{G}$ in different solvents. Spectral half-widths are again noted in gauss units. 
FR2 arises from a partially reversible change of a laser-excited dye molecule.

Finally, in order to determine the connection of the amount of $\mathrm{R} 6 \mathrm{G}$ molecules in the $T_{1}$ state with the producing processes of those radicals we examined the relation between the molecular concentration before the photochemical reaction and the radical concentration after the reaction for the $\mathrm{R} 6 \mathrm{G}$-ethanol solution, adding different amounts of a COT quencher. The result, which is shown in Figs. 9 and 10 , is that the radical concentration (for the FR1 we got the same values during and after the UV laser irradiation and, for the FR2, different values during and after the $5145-\AA$ laser irradiation) decreases as the concentration of the molecules in the $T_{1}$ state before the reaction decreases. This shows that the molecules in the $T_{1}$ state have direct effects upon those two laser-induced chemical reactions. In particular, the FR2 appearing during or after the $5145-\AA$ laser irradiation decreases even more rapidly than the dye molecule in the $T_{1}$ state decreases due to the quenching by an energy transfer to a COT molecule. From this we conclude that the FR2, which depends on the chromophoric

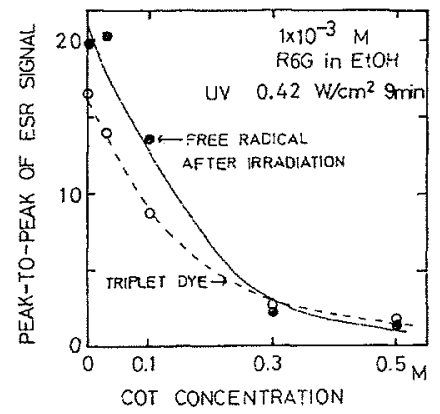

Fig. 9. Dependences of the peak-to-peak heights of the $H_{\text {min }}$ line before the radicals occurred (symbol $O$ ) and of the radical spectrum induced after an UV laser irradiation (symbol $\bullet$ ) on the COT concentration in an R6G-ethanol solution at $77 \mathrm{~K}$. The $H_{\min }$ line was recorded in the same way as in the case of Fig. 2 .

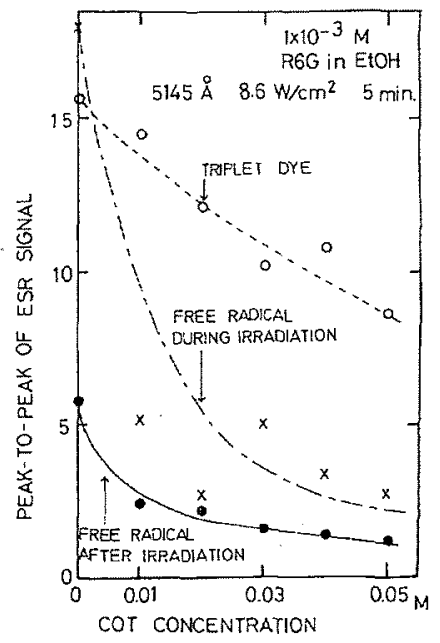

Fig. 10. Dependences of the peak-to-peak heights of the $H_{\min }$ line befote the radicals occurred (symbol o) and of the radical spectrum induced during (symbol $X$ ) or after (symbol $\bullet$ ) a 5145- $\AA$ laser irradiation on the COT concentration in an R6G-ethanol solution at $77 \mathrm{~K}$. The $H_{\mathrm{min}}$ line was recorded in the same way as in the case of Fig. 2. structure of the dye, is produced by a partially reversible change of one dye molecule in the $T_{1}$ state.

\section{Conclusion}

Based on our considerations, we find that the mechanisms of the photodegradations occurring in R6G-ethanol laser solutions at $77 \mathrm{~K}$ are quite different for the two cases of UV and 5145-A laser excitations, and the laser-induced photochemical reactions are explained in the following ways:

A) in the case of an UV laser irradiation including flashlamp excitation;

1) a few of the dye molecules excited to the $S_{1}$ state by the absorption of a photon decay to the $T_{1}$ state by an intersystem crossing process;

2) some of them return to the $S_{0}$ state, but others absorb another photon to produce a higher excited triplet state ( $T-T$ absorption);

3) the $\mathrm{C}-\mathrm{H}$ bonds of the ethanol are ruptured by an energy transfer from a dye molecule in the higher triplet state to a solvent molecule, which results in the FR1 radical, and the dye is reduced to a leuco compound [12]:

B) in the case of a 5145- $A$ laser irradiation;

1) like A) 1), some dye molecules are excited to the $T_{1}$ state;

2) except for decaying to the $S_{0}$ state, the dye molecule in the $T_{\mathfrak{l}}$ state changes in to another radical, FR2.

The schemes of the proposed reaction sequences in both cases A) and B) are shown in Fig. 11. Thus we think that one of the most important procedures to increase the lifetime of xanthene dye lasers is to use a triplet quencher and an unreduc-

PROPOSED PHOTODEGRADATION MECHANISM

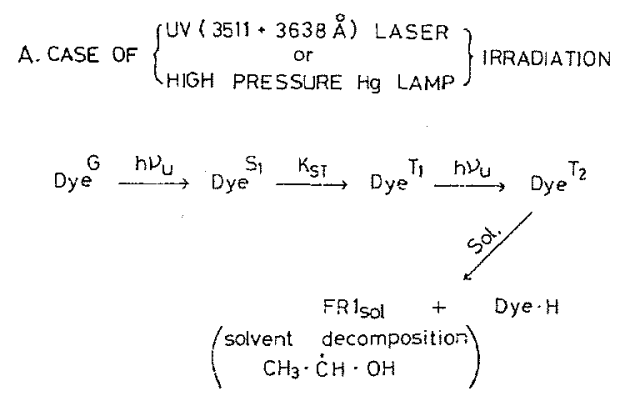

B. CASE OF $5145 \AA$ A LASER IRRADIATION

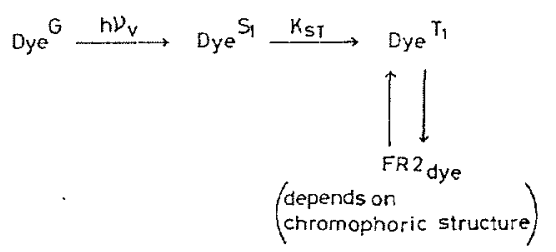

Fig. 11. Proposed photodegradation mechanisms occurring in a laser dye solution under UV laser or flashlamp excitations [case A)] and 5145-A laser excitation [case B)] . 
ible solvent. Finally, it should be pointed out that there are still some problems to be solved. Mainly, what is the exact nature of the second reaction $B$ ) resulting in the FR2 radicals? But the information necessary to answer this question is not yet available.

\section{ACKNOWLEDGMENT}

The authors wish to thank Dr. K. Sakurai and Dr. T. Sato for their continuous encouragement. They also wish to thank H. Ikeda for his help with the experimental work.

\section{REFERENCES}

[1] E. P. Ippen, C. V. Shank, and A. Dienes, "Rapid photobleaching of organic laser dyes in continuously operated devices," IEEE J. Quantum Electron. (Corresp.), vol. QE-7, pp. 178-179, Apr. 1971.

[2] A. D. Britt and W. B. Moniz, "The effect of $\mathrm{pH}$ on photobleaching of organic laser dyes," IEEE J. Quantum Electron. (Corresp.), vol. QE-8, pp. 913-914, Dec. 1972.

[3] J. Weber, "Study of the influence of triplet quencher an the photobleaching of thodamine 6G," Opt. Commun., vol. 7, pp. 420-422, Apr. 1973.

[4] M. Yamashita and H. Kashiwagi, "Triplet-state esr of rhodamine 6 G during laser irradiation," J. Chem. Phys., vol. 59, pp. 21562157, Aug. 1973.
[5] R. A. Berg and A. Ron, "Phosphorescence lifetimes of cationic dyes: Concentration and temperature effects," J. Chem. Phys., vol. 59, pp. 3289-3296, Aug. 1973.

[6] M. Yamashita and H. Kashiwagi, "Temperature effects on triplet lifetime of thodamine $6 \mathrm{G}$ in ethanol and polymethylmethacrylate," Japan. J. Appl. Phys., vol. 14, pp. 421-422, Mar. 1975.

[7] - "Optical saturation and quenching effects in the triplet state of rhodamine 6G," J. Phys. Chem., vol, 78, pp. 20062009 , Sept. 1974

[8] M. Yamashita, H. Ikeda, and H. Kashiwagi, "Laser pumped magnetophotoselection in triplet dyes," J. Chem. Phys., vol. 63, pp. 1127-1132, Aug. 1975.

[9] J. F. Gibson, M. C. R. Symons, and M. G. Townsend, "Unstable intermediates. Part II. Photolysis of hydrogen peroxide in solid alcohols: Some reactions of hydroxyl radicals," $J$. Chem. Soc. pp. 269-276, 1959.

[10] K. Shimokoshi, Y. Mori, and I. Tanaka, "An esr study of the aromatic-compounds-photosensitized decomposition of alcohol by the two-photon process," Bull. Chem. Soc. Japan, vol. 40 , pp. 302-306, Feb. 1967.

[11] S. Siegel and K. Eisenthal, "Triplet-triplet absorption in sensitized solvent decomposition: An esr study," J. Chem. Phys., vol. 42, pp. 2494-2502, Apr. 1965.

[12] K. Uchida, S. Kato, and M. Koizumi "Photosensitized oxidation of leuco-uranine and the identification of the photobleached product of uranine in ethanol solution," Bull. Chem. Soc. Japan, vol. 35 , pp. 16-22, Jan. 1962 . 